\title{
Storage ring probes of dark matter and dark energy
}

\author{
Peter W. Graham $\odot,{ }^{1}$ Selcuk Hacıömeroğlu $\odot,{ }^{2}$ David E. Kaplan, ${ }^{3}$ Zhanibek Omarov $\odot,{ }^{4,2}$ \\ Surjeet Rajendran, ${ }^{3}$ and Yannis K. Semertzidis $\oplus^{2,4}$ \\ ${ }^{1}$ Stanford Institute for Theoretical Physics, Department of Physics, \\ Stanford University, Stanford, California 94305, USA \\ ${ }^{2}$ Center for Axion and Precision Physics Research, Institute for Basic Science, \\ Daejeon 34051, Republic of Korea \\ ${ }^{3}$ Department of Physics \& Astronomy, The Johns Hopkins University, \\ Baltimore, Maryland 21218, USA \\ ${ }^{4}$ Department of Physics, Korea Advanced Institute of Science and Technology, \\ Daejeon 34141, Republic of Korea
}

(Received 26 November 2020; accepted 24 January 2021; published 15 March 2021)

\begin{abstract}
We show that proton storage ring experiments designed to search for proton electric dipole moments can also be used to look for the nearly dc spin precession induced by dark energy and ultralight dark matter. These experiments are sensitive to both axion-like and vector fields. Current technology permits probes of these phenomena up to 3 orders of magnitude beyond astrophysical limits. The relativistic boost of the protons in these rings allows this scheme to have sensitivities comparable to atomic comagnetometer experiments that can also probe similar phenomena. These complementary approaches can be used to extract the microphysics of a signal, allowing us to distinguish between pseudoscalar, magnetic and electric dipole moment interactions.
\end{abstract}

DOI: $10.1103 /$ PhysRevD.103.055010

\section{INTRODUCTION}

A variety of cosmological and astrophysical measurements have established that nearly $95 \%$ of the energy density in the Universe today is in dark energy and dark matter. The nature of these cosmic fluids remains a mystery. Since these fluids form a preferred (cosmic) background, a generic way to search for the properties of these fluids is to look for the effects of the motion of standard model particles against this cosmic background "ether." Experiments that test Lorentz invariance can thus be reinterpreted as searches for interactions between the standard model and these dark fluids [1].

Spin precession experiments [2-6] are a canonical test of Lorentz invariance. These look for an anomalous precession of a spin caused by an interaction between a spin moving against a background classical field. These experiments are a particularly well motivated way to search for ultralight dark matter candidates such as axions and axion-like particles, which naturally possess such spinprecession-inducing interactions [7]. Moreover, unlike dark

Published by the American Physical Society under the terms of the Creative Commons Attribution 4.0 International license. Further distribution of this work must maintain attribution to the author(s) and the published article's title, journal citation, and DOI. Funded by SCOAP ${ }^{3}$. matter where ultralight particles are simply a possible class of dark matter candidates, any viable dark energy candidate that is not a cosmological constant has to be an ultralight classical field. Axion-like interactions that induce spin precession are also a natural expectation of such dark energy candidates, particularly when those candidates can actually solve the cosmological constant problem $[8,9]$.

Spin precession experiments that search for axion dark matter [10] are largely focused on axions with a mass larger than $\sim 1 \mathrm{~Hz}$. In this regime, many technical sources of noise (such as vibrations and magnetic shielding) are more easily ameliorated. There is however considerable scientific motivation to develop techniques that can search for such signals at much lower frequencies. This is important since the observational limit on the mass of dark matter is as low as $10^{-7} \mathrm{~Hz}$, wherein dark matter in this mass range gives rise to an essentially dc signal in an experiment. Moreover, the spin precession induced by dark energy will also be a dc signal since any change to the frequency of this signal has to be comparable to the Hubble scale $\sim 10^{-18} \mathrm{~Hz}$. The challenges of a dc spin precession signal are currently combated through the use of atomic comagnetometers $[2,4-6]$, where the relative precession between two species in a medium is used to cancel out many sources of technical noise.

In this paper, we propose the use of storage rings as an alternate technique to search for such spin precession. Storage rings are devices where a relativistic beam of 
particles (for concreteness, we will consider protons in this paper) are stored for significant periods of time by means of electric and/or magnetic fields. These rings possess a magic momentum wherein the precession of a spin in the electromagnetic fields of the ring is compensated by the motion of the particle around the ring thus maintaining a fixed orientation of the spin relative to the direction of motion of the particle. By measuring the spin of the particles, these storage rings can search for anomalous precession. These rings have been used to search for fundamental electric dipole moments (EDMs) [11,12]. The notion of the magic momentum was first invented at the third CERN muon $g-2$ experiment, where the muon spin precession frequency is not affected by the presence of the electric focusing system, while its $g-2$ frequency can be measured with high accuracy in a highly uniform $B$ field [13].

Inspired by these designs, we evaluate the feasibility of storage rings as a way to search for ultralight dark matter and dark energy. We will show that storage rings can have sensitivities comparable to atomic comagnetometer techniques for pseudoscalar interactions. For vector backgrounds, due to the relativistic nature of the beam, these rings have enhanced sensitivity to magnetic dipole interactions and can thus distinguish between electric and magnetic dipole interactions. Storage ring techniques are thus complementary to atomic comagnetometer searches: the combination of both techniques can be used to extract the underlying nature of any new physics discovered in such experiments. The rest of this paper is organized as follows. In Sec. II, we present a theoretical overview of the signals of dark energy and dark matter in a storage ring. In Sec. III, we discuss the storage ring setup and evaluate the sensitivity of this approach. Following this, we discuss experimental backgrounds and ways to ameliorate them to the required levels in Sec. IV. We conclude in Sec. V, where we also compare and contrast this approach to atomic comagnetometer searches in greater detail.

\section{THE THEORY OF THE EFFECT}

For the purposes of this article, we are interested in extremely light (pseudo)scalar fields coupled to protons. Such a coupling can come from an underlying coupling to quarks, e.g.,

$$
\mathcal{L} \supset g_{a q q} \partial_{\mu} a \bar{q} \gamma^{\mu} \gamma_{5} q \rightarrow g_{a N N} \partial_{\mu} a \bar{p} \gamma^{\mu} \gamma_{5} p
$$

where $a$ is the light spinless field, and $q$ is a light quark and $p$ is the proton. Such quark couplings can come from spontaneous breaking of a global symmetry at high scales. Assuming such a symmetry is anomaly free with respect to QCD, the "axion," $a$, does not receive an instanton-induced mass and will remain naturally light.

In the rest frame of the proton, this term generates the effective Hamiltonian term:

$$
H \ni-g_{\mathrm{aNN}} \vec{\nabla} a \cdot \vec{\sigma}
$$

where $\sigma$ is the proton spin. Thus, the gradient of the axion field couples to the spin of the proton like a magnetic field. The physical effect is the precession of the proton spin around the vector $\vec{\nabla} a$. The size of the gradient of the axion field is set by the axion momentum: $\vec{\nabla} a \sim \gamma m_{a} \beta a$ where $m_{a}$ is the axion mass and $\beta$ is its velocity in the proton rest frame and $\gamma$ is the relativistic gamma factor.

Here is where the storage ring is interesting. A laboratory experiment is moving at a velocity $\beta \sim 10^{-3}$ relative to the virial velocity of dark matter or the rest frame of dark energy, either of which could have an axion component. On the other hand, in a storage ring experiment at relativistic velocities, we get an automatic enhancement with $\beta \sim \mathcal{O}(1)$. The $\gamma$ factor however cancels due to time dilation, as the integration time is measured in the lab frame.

\section{A. Dark energy}

In the case of dark energy, we imagine a field $a$ rolling down a shallow potential with a nonzero $\partial_{t} a$, homogeneous in the rest frame of the cosmic microwave background (CMB). Taking the kinetic energy of $a$ to be a fraction $\epsilon$ of the dark energy density $\rho_{\mathrm{DE}}$, we have (in the proton rest frame)

$$
\partial_{\mu} a=\left\{\gamma \sqrt{2 \epsilon \rho_{\mathrm{DE}}}, \gamma \vec{\beta} \sqrt{2 \epsilon \rho_{\mathrm{DE}}}\right\}
$$

and thus the proton's spin will couple to the axion gradient analogous to how a magnetic field couples to its magnetic moment. A proton spin not aligned with the direction of the velocity will precess around $\vec{\beta}$ at a precession frequency of $\nu_{\text {prec }} \simeq g_{\mathrm{aNN}} \beta \sqrt{2 \epsilon \rho_{\mathrm{DE}}} / 2 \pi$ in cycles per second in the lab frame. Note that for a proton traveling in a straight line, the effect always rotates the spin around the same direction around the velocity, and thus the effect adds over long integration times. Thus, after a time $T$, the precession angle would be $\Delta \theta_{\text {prec }} \simeq g_{\mathrm{aNN}} \beta \sqrt{2 \epsilon \rho_{\mathrm{DE}}} T$. The size of the effect will be set by the coupling $g_{\mathrm{aNN}}$, the experimental value of dark energy density is $\rho_{\mathrm{DE}} \sim\left(2 \times 10^{-3} \mathrm{eV}\right)^{4}$, and the fraction of dark energy in the axion's kinetic energy (which we define as the parameter ?). The current bound on $\epsilon$ can be loosely extracted from the Planck Collaboration's fit to dynamical dark energy: $\epsilon \simeq(w+1) / 2<0.06(0.20)$ at $1 \sigma(2 \sigma)$, where $w$ is the dark energy equation of state measured today [14].

\section{B. Dark matter}

\section{Pseudoscalars}

In the case of $a$ dark matter, the main difference with dark energy is the fact that the $a$ field value (and thus the time derivative) oscillates in time as $a \sim \frac{\sqrt{2 \rho_{\mathrm{DM}}}}{m_{a}} \sin \left(m_{a} t\right)$, 
where $\rho_{\mathrm{DM}}$ is the local dark matter energy density. From time $t$ to $d t$, the proton spin precesses an amount $d \theta_{\text {prec }} \simeq g_{\mathrm{aNN}} \beta \sqrt{2 \rho_{\mathrm{DM}}} \cos \left(m_{a} t\right) d t$. Thus, in the case of dark matter, the precession angle after a time $T$ would be

$$
\left.\Delta \theta_{\mathrm{prec}} \simeq \frac{g_{\mathrm{aNN}} \beta \sqrt{2 \rho_{\mathrm{DM}}}}{m_{a}} \sin \left(m_{a} T\right)\right)
$$

in the lab frame. For $m_{a} \ll 1 / T$, the effect again adds coherently, whereas for $m_{a} \gtrsim 1 / T$, the precession angle oscillates in time.

\section{Vectors}

We also note that spin precession of the proton would also result if the dark matter were a light vector, $A_{\mu}^{\prime}$. Consider the magnetic dipole moment and electric dipole moment operators, which couple the vector (hidden photon) field to protons:

$$
\mathcal{L} \supset g_{\mathrm{MDM}}^{\prime} F_{\mu \nu}^{\prime} \bar{p} \sigma^{\mu \nu} p+g_{\mathrm{EDM}}^{\prime} F_{\mu \nu}^{\prime} \bar{p} \gamma^{5} \sigma^{\mu \nu} p
$$

where $F_{\mu \nu}^{\prime}=\partial_{\mu} A_{\nu}^{\prime}-\partial_{\nu} A_{\mu}^{\prime}$. Under the same assumption as in the axion case that the hidden (dark) photon field can be modeled as a classical wave, we can estimate the amplitude of the oscillating dark electric field as $E_{\text {lab }}^{\prime} \simeq \sqrt{\rho_{\mathrm{DM}}}$ in the rest frame of the $\mathrm{CMB}$, while the dark magnetic field is velocity suppressed in this frame. In the frame of a proton traveling at a relativistic velocity $\vec{\beta}$, the dark magnetic field is $\vec{B}^{\prime}=\vec{\beta} \times \vec{E}^{\prime}$, relative to the dark electric field in the same frame. In this frame, the spin of the proton will precess around the $B$ and $E$ fields due to the magnetic dipole moment (MDM) and EDM operators respectively.

For a proton traveling in a circular ring, a dark $E$ field in the lab frame, perpendicular to the plane of the ring generates a dark $B$ field pointing radially. For the MDM operator, a spin locked in the direction of the velocity $\vec{\beta}$ will precess out of the ring's plane. An $E$-field component in the ring's plane will cause precession in the plane of the ring that oscillates. Such precession will thus cancel over each round trip of the proton. Thus, if the dark electric field is pointing at an angle $\phi$ with respect to the ring plane's normal, a proton spin initially parallel to the velocity will have a precession angle after a time $T$ of

$$
\Delta \theta_{\mathrm{prec}} \simeq \frac{g_{\mathrm{MDM}}^{\prime} \beta \sqrt{\rho_{\mathrm{DM}}}}{m_{A^{\prime}}} \cos \phi \sin \left(m_{A^{\prime}} T\right),
$$

where we have ignored the in-plane oscillating precession by assuming an integer number of trips around the ring. The $\cos \phi$ picks out the normal component of the $E$ field, and $m_{A^{\prime}}$ is the dark photon mass.

Aligning the spin parallel with the velocity, the EDM operator will cause a similar precession due to a perpendicular dark $E$ field, but this time in the plane of the ring. One can get precession out of the plane due to a coplanar dark $E$ field. If the spin is kept in a fixed planar direction in the lab frame, then the dark planar $E$ field will produce a precession out of the plane after a time $T$

$$
\Delta \theta_{\mathrm{prec}} \simeq \frac{g_{\mathrm{EDM}}^{\prime} \sqrt{\rho_{\mathrm{DM}}}}{m_{A^{\prime}}} \sin \phi \sin \left(m_{A^{\prime}} T\right),
$$

where $\phi$ again is the angle between the dark $E$ field and the plane's normal.

An interesting aspect of this signal is that its magnitude should have a daily modulation as the plane of the ring evolves with the Earth's and the angle $\phi$ oscillates with a 24 hour period. This will be a modulation on top of the main oscillation of the dark matter at frequency $m_{A^{\prime}}$

\section{A STORAGE RING EXPERIMENT}

The spin precession caused by the axion wind effect is proportional to the gradient of the axion field, as seen above. For a lab experiment searching for dark matter or dark energy this effect would usually be suppressed by the low velocity of the axion field $\left(\sim 10^{-3}\right.$ for the relative velocity between the Earth and either the dark matter or dark energy). However if we boost the precessing particle up to relativistic velocities then the effect is significantly increased. While this is of course not possible for most sensitive spin precession experiments, there is one type of sensitive experiment with relativistic spins: a storage ring experiment such as a muon $g-2$ or proton EDM measurement (see e.g., Ref. [12]). As we will see, the proton storage ring EDM proposal can indeed be used to search for axion dark matter and dark energy.

When a proton is boosted to relativistic speeds it sees a much larger spatial gradient of the axion field directed along the direction of the boost. The proton's spin will then precess around this direction. Of course in a storage ring the velocity sweeps in a circle and so the net precession would generally cancel out (or be significantly reduced) as the proton goes around the ring many times. To fix this we can use the "frozen spin" method where the spin of the proton is always locked to a fixed angle with respect to the velocity (see Fig. 1). Then the effect will add up over the entire orbit of the proton around the ring. In this way we will boost the signal and be able to add it up over the entire integration time of the storage in the ring ( $1000 \mathrm{~s}$ for the proton EDM experiment).

The proton storage ring EDM proposal uses this "frozen spin" method $[12,15]$. In that proposal the proton is placed in a ring with a large electric field (either all electric or a hybrid electric-magnetic design) and its spin is aligned with its velocity. The proton is given the "magic momentum" so that the ring's electromagnetic fields cause the spin to precess by $2 \pi$ in exactly the time the proton orbits the ring once, thus keeping the spin and velocity always aligned. This can be seen easily in the proton's rest frame where the 

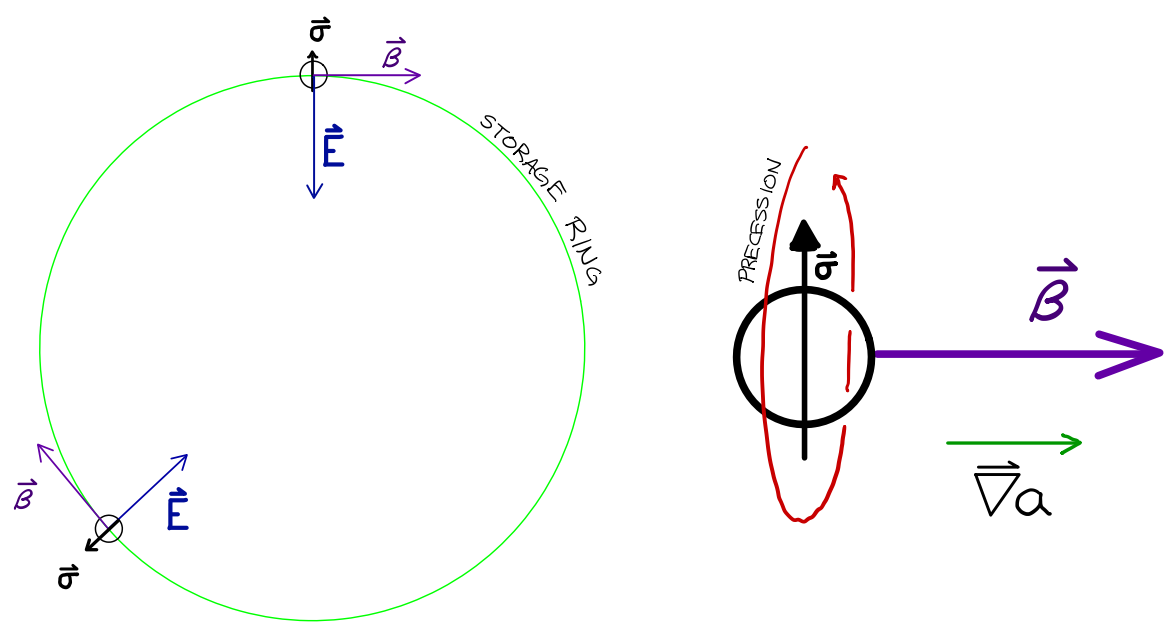

FIG. 1. A sketch of the geometry for this storage ring proposal (left figure) and the directions of the proton's spin $\vec{\sigma}$, velocity $\vec{\beta}$ and precession, as well as the axion field gradient seen by the proton (right figure). The proton's spin must be oriented radially and will then precess around its velocity (out of the plane of the ring).

large radial electric field looks like it has a large magnetic component perpendicular to the plane of the ring. If the proton has an EDM, then its spin will also precess around the large electric field and thus out of the plane of the ring. The protons spins are measured continuously over the period of about $1000 \mathrm{~s}$ that they spend in the ring.

Our proposal is to use the same storage ring to search for time-varying dark energy (with axionic couplings) and axion (or vector) dark matter. Note that for the axion case, as in Fig. 1, the proton's spin must be oriented radially (instead of tangentially as in the EDM case) so that it will precess around the proton's velocity, out of the plane of the ring. The signal of axion dark matter or dark energy then is a small rising component of the proton's spin out of the plane as a function of time. Thus the same storage ring can be used to search for dark matter and dark energy as will be used for the proton EDM. We are just searching for a different signal with a different dependence on the spin orientation of the proton and also, in the case of dark matter, with a fixed temporal frequency (see Sec. II).

In Fig. 2 we show an estimate for the sensitivity of this proposal to axion dark matter. Figure 3 shows the sensitivity to time-varying dark energy, assuming it has an axion-like coupling. We have assumed similar numbers to

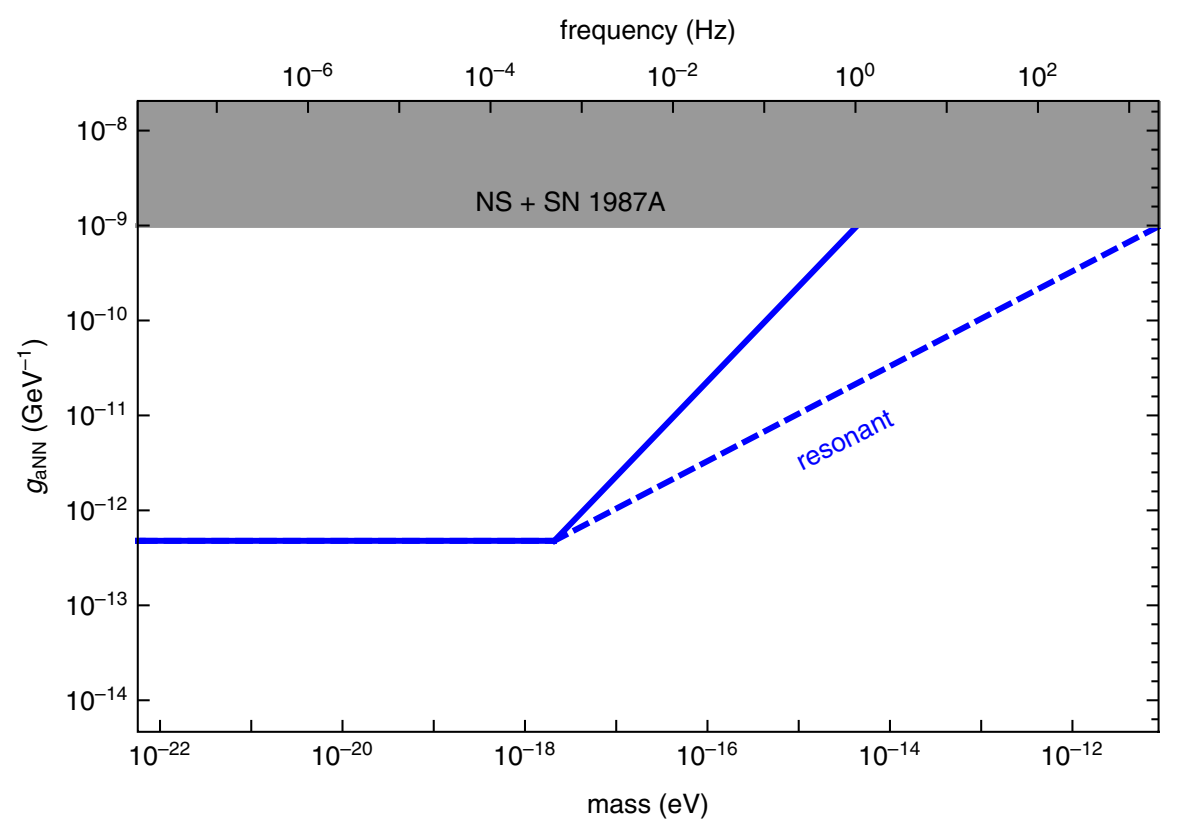

FIG. 2. The sensitivity of the storage ring proposal to axion dark matter in axion-nucleon coupling $g_{\mathrm{aNN}}$ vs mass of axion. The gray region is excluded by excess cooling in neutron stars and SN1987A. The solid blue line shows the estimated sensitivity of the proton storage ring experiment assuming a $1000 \mathrm{~s}$ storage time and sensitivity to a proton spin precession rate of $10^{-9} \mathrm{rad} / \mathrm{s}$. The dashed blue line shows the sensitivity of a resonant version of the experiment. 


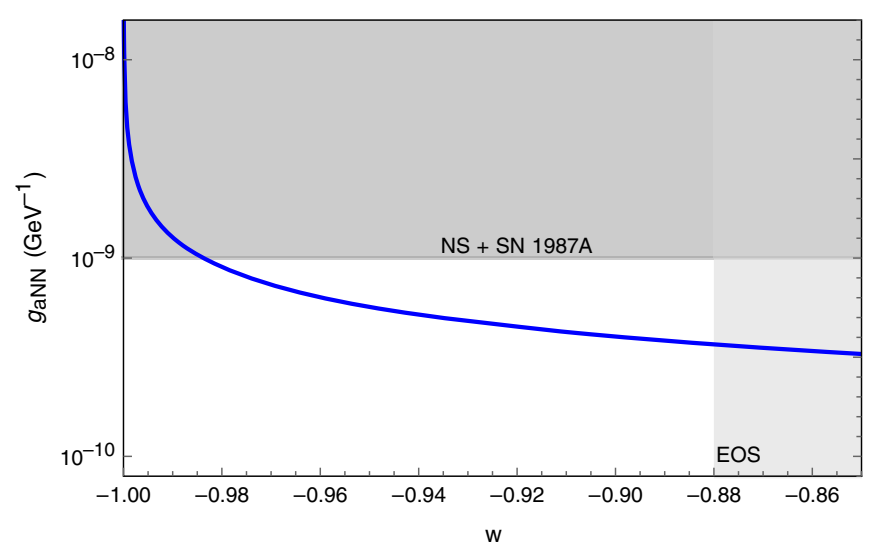

FIG. 3. The sensitivity of this storage ring experiment to timevarying dark energy with an axion-nucleon coupling $g_{\mathrm{aNN}}$ vs equation of state of dark energy $w$. The gray region is excluded by excess cooling in neutron stars and SN1987A. The solid blue line shows the projected sensitivity of the proton storage ring experiment assuming a $1000 \mathrm{~s}$ storage time and sensitivity to a proton spin precession rate of $10^{-9} \mathrm{rad} / \mathrm{s}$.

the storage ring EDM proposal, namely the spin coherence time of the proton beam in the ring is $1000 \mathrm{~s}$ and that overall the sensitivity of the entire experiment after all protons have been sent through the ring will allow signals as small as a proton spin precession rate of $10^{-9} \mathrm{rad} / \mathrm{s}$ out of the plane of the ring to be measured. For simplicity we have enveloped the sensitivity curve to remove the spikes that come from having a fixed time of $1000 \mathrm{~s}$. This could in practice be achieved by varying this time by an order 1 factor from shot to shot.

The current astrophysical bounds on the axion-nucleon (really we consider only the axion-proton) coupling come from excess cooling of supernovae and neutron stars [1619]. While there is some uncertainty in these due to modeling of the astrophysical object, these bounds cannot move too far and we have quoted an average value.

For axion dark matter frequencies above $\sim(1000 \mathrm{~s})^{-1}$ this experiment loses sensitivity because the axion signal averages out over the time each proton spends in the ring. We could gain back some of this sensitivity by doing a resonant search. If the proton momentum is tuned slightly away from the magic momentum then the spin will precess at a rate slightly different than the rate at which velocity is rotating. The difference in the spin precession frequency and the frequency with which the velocity changes direction (which is the orbital frequency) is then the resonant frequency of this experiment. By changing the detuning from the magic momentum, the resonant frequency can be swept to search for the axion. The sensitivity of this resonant technique is shown as the dashed line in Fig. 2.

The Muon $g-2$ Experiment at Fermilab is also a storage ring experiment and could in principle be used to search for axion dark matter with a coupling to muons, though since the spin is not frozen the sensitivity would be dominantly

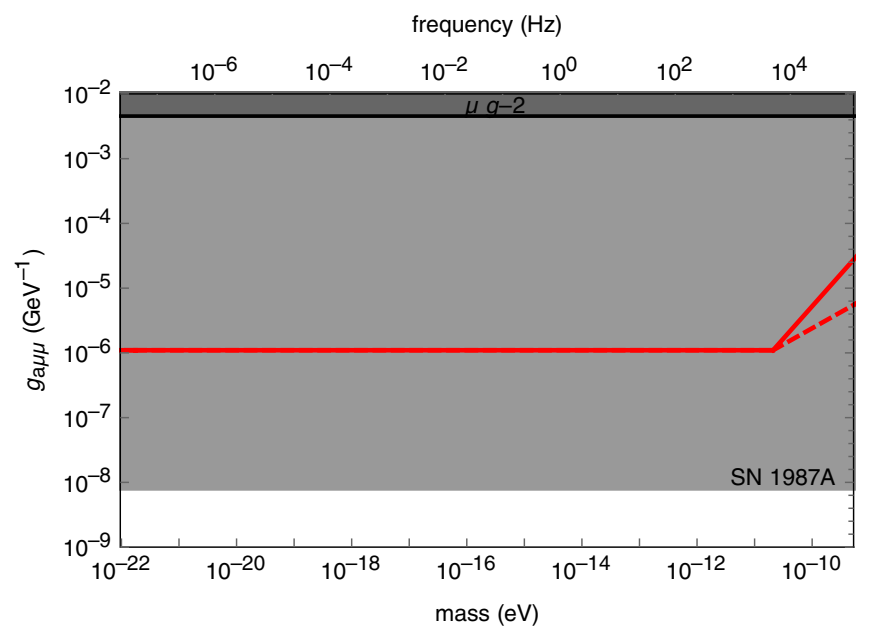

FIG. 4. The sensitivity of the storage ring proposal (with muons) to axion dark matter with an axion-muon coupling $g_{\mathrm{a} \mu \mu}$ vs mass of axion. The gray region is excluded by excess cooling in SN1987A [20]. The dark gray region is the best current laboratory bound coming from virtual axion contributions to the muon $g-2$ [21]. The solid red line shows the estimated sensitivity of the storage ring experiment with muons. The experimental parameters assumed are detailed in the text. The dashed red line shows the sensitivity of a resonant version of the experiment. The current Muon $g-2$ Experiment at Fermilab would have a sensitivity a little over an order of magnitude worse than the red line over most of frequency space.

only in one narrow frequency band. Muons could also in principle be placed in the same proton EDM storage ring. In this case the muons' spins could be frozen and we could have sensitivity to a wide range of dark matter frequencies (and also to dark energy). We show the estimated sensitivity for such a dark matter search in Fig. 4. As example parameters for such an experiment with muons we have assumed a total sensitivity to an axion-induced muon precession rate of $2.3 \frac{\mathrm{mrad}}{\mathrm{s}}$ in the ring. Since the Fermilab $g-2$ experiment does not freeze the muons' spins relative to their velocity, the total sensitivity of that experiment ends up being a little over a factor of 10 worse than the dedicated experiment. Of course the Fermilab experiment currently has data and could do such an analysis now which would already give the best laboratory bound on the axion-muon coupling.

The strongest current limit on the axion-muon coupling comes from SN1987A [20]. There are also bounds from the number of degrees of freedom produced in the early Universe, $N_{\text {eff }}[22,23]$ which are not currently as constraining but will improve significantly with CMB S4 [24] (and see the approximate CMB bound in Ref. [20]). The strongest laboratory bound comes from the virtual axion contributing to the muon $g-2$, which we quote as the " $\mu g-2$ " region in Fig. 4 [21].

Finally, we note that for vector dark matter and the magnetic dipole operator, the coupling $g_{\mathrm{MDM}}^{\prime}$ can be 
obtained by using the results of Fig. 2 and setting $g_{\mathrm{MDM}}^{\prime}=2 g_{\mathrm{aNN}}$. The factor of 2 comes from a combination of normalization as well as averaging over the $\cos \phi$, the angle between the dark $E$ field and the normal to the ring (though the daily modulation of this signal adds an interesting effect).

The electric dipole operator could be effectively tested if the spin of the orbiting particle were frozen in the lab frame. Such a setup would be possible with a particle like the deuteron, which has a negative anomalous magnetic moment. The change in the particle spin in the lab frame is

$$
\begin{aligned}
\frac{d \vec{\sigma}}{d t}= & \frac{e}{m} \vec{\sigma} \times\left[\left(a+\frac{1}{\gamma}\right) \vec{B}-\frac{a \gamma}{\gamma+1} \vec{\beta}(\vec{\beta} \cdot \vec{B})\right. \\
& \left.-\left(a+\frac{1}{\gamma+1}\right) \vec{\beta} \times \vec{E}\right]
\end{aligned}
$$

where $e / m$ is the charge-to-mass ratio and $a=(g-2) / 2$ is the anomalous magnetic moment. We see for a storage ring with a radial electric field, the spin stays fixed if $\gamma=-(1 / a)-1$. For a ring with a perpendicular magnetic field, we require $\gamma=-(1 / a)$. For deuterium $(a \simeq-.14)$, these gamma factors are roughly 6 and 7 respectively.

\section{EXPERIMENTAL SETUP AND BACKGROUNDS OVERVIEW}

The experiment for the most part is described in Ref. [12] where an all-electric ring is assumed, with some very important differences shown here. In that case, the bending is provided by a pair of vertical cylindrical plates to accommodate the particle bending horizontally and the focusing is provided by electrostatic quadrupoles. The main challenge in this experiment is its sensitivity to the unwanted magnetic fields that inevitably would be present, requiring novel methods of detecting them. The technology to accomplish the required level of cancellation has been developed [25]. The hybrid version [26] of the method, however, reduces it automatically by several orders of magnitude via alternate magnetic, instead of electric focusing. In general, the main ring focusing method also determines the nature of the prominent systematic error. In rings with electric focusing, the main systematic error is unwanted magnetic fields, while in rings with magnetic focusing the main systematic error is an out-of-plane electric field. When the effect of electric forces on beam dynamics is balanced by magnetic force or vice versa, an unwanted vertical spin precession is created. In addition, the compensated gravitational forces also induce a significant vertical spin precession that can only be resolved by $\mathrm{CW}$ and CCW storage [27]. A stored particle is assured to feel a total vertical force equal to zero by definition, but the delicate balance of those forces is critical. The effect of those forces on the vertical spin precession rate depends on the particle's velocity and spin direction and in general it is different than when they balance each other in beam dynamics. In general, they do not balance in spin dynamics nor by subtracting the effects from clockwise $(\mathrm{CW})$ and counterclockwise $(\mathrm{CCW})$ beams, and thus they present a serious systematic error source. Even though the gravity effect cancels comparing the $\mathrm{CW}$ and $\mathrm{CCW}$ effects, and in practice it is not an issue, the balancing act of combined electric and magnetic fields is more complicated and presents a serious challenge. Both focusing cases listed above are very challenging to achieve, i.e., either the required cancellation of unwanted magnetic fields or the alignment of the electric fields in the bending sections. However, in the hybrid version where alternate magnetic focusing is applied, it is also possible to store beams simultaneously traveling $\mathrm{CW}$ and $\mathrm{CCW}$, which exactly cancels all out-of-plane dipole electric fields, the main systematic error source in this case. Cancellation of higher than dipole order electric fields require $\mathrm{CW}-\mathrm{CCW}$ beam closed orbits to trace the same path around the ring to $0.01 \mathrm{~mm}$. Therefore, the claim is that the hybrid lattice ring is in general easier and cheaper to achieve and this is what we will assume for the rest of the paper.

In the storage ring proton EDM experiment the beam is bunched by a radio-frequency (RF) cavity inducing the so-called synchrotron oscillations [28,29] for the captured protons. Within the bunch, the proton momentum oscillates with an amplitude depending on its momentum deviation from the average momentum and its relative arrival time at injection. The RF cavity is designed to equalize, to first order, all the proton momenta to the desired momentum value. For one such momentum value, the so-called "magic" momentum, given by $p=m c / \sqrt{G} \approx$ $0.7 \mathrm{GeV} / \mathrm{c}$ for the proton, where $m$ is the proton mass, $c$ is the speed of light and $G=\left(g_{p}-2\right) / 2=1.792847356$ is the proton anomaly, the relative angle in the horizontal plane between the proton spin and momentum vectors will remain "frozen" if the horizontal bending is caused by only electric fields. [12] There are two main reasons for applying the RF cavity in the proton EDM experiment. It increases the spin coherence time of beams, e.g., with $d p / p \approx 10^{-4}$ from the milliseconds range without it to several seconds. $[12,30,31]$ In addition, it is essential in eliminating the polarimeter systematic errors [32] arising from a potential beam motion in position and angle relative to the external target and detector system. The spin coherence time can be further increased by the addition of sextupoles at an appropriate place $[12,30,31,33]$ in the ring and/or by reducing the captured beam phase space at injection. A single RF cavity can capture both $\mathrm{CW}$ and $\mathrm{CCW}$ traveling particles. ${ }^{1}$ The number of bunches captured in the EDM

\footnotetext{
${ }^{1}$ The RF cavity operates in the $\mathrm{TM}_{010}$ mode with the electric field in the longitudinal direction and a resonant frequency that is a multiple of the proton revolution frequency. In a regular storage ring, only half the RF cycle captures particles in stable synchrotron oscillations. The counterrotating beams are captured in the opposite cycles matching the direction of motion.
} 


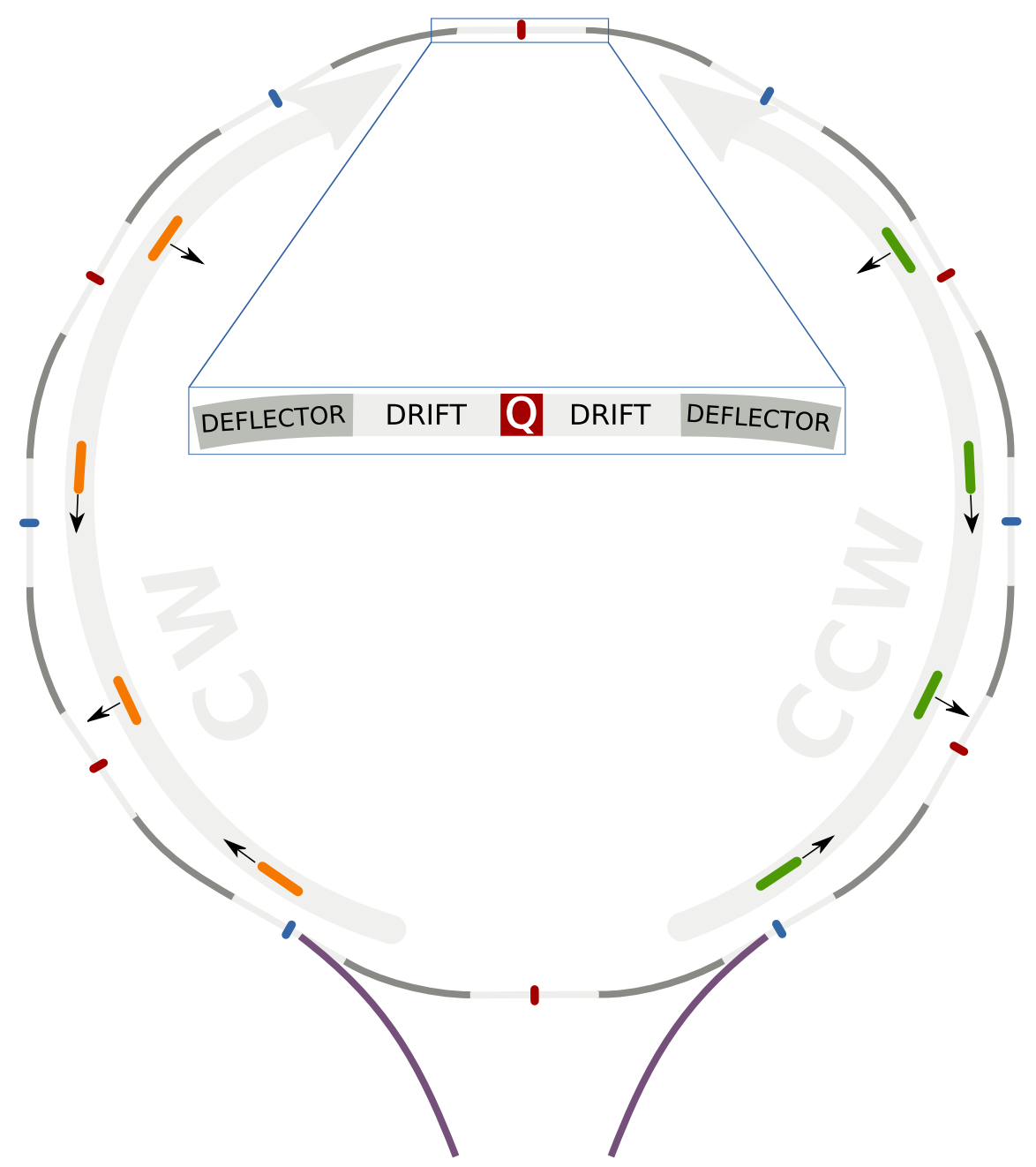

FIG. 5. The ring lattice is symmetric for $\mathrm{CW}$ and $\mathrm{CCW}$ beams, but also with respect to each lattice element in the ring. The ring lattice parameters are given in Table I. This design has been shown to be more robust against lattice-element position tolerances since the particle deviations from its ideal orbit cancel to a high degree, when integrated around the ring. The beam bunch polarization is also indicated, showing that all four spin directions are simultaneously stored for both $\mathrm{CW}$ and CCW beams for systematic error cancellations.

ring is of the order of $10^{2}$, while the polarization direction in half the bunches is kept in the longitudinal direction and of those, half of them have positive and the other half have negative helicity. The remaining half of the bunches have the proton spin oriented in the radial direction half of which again are pointing radially outward and half inward, ${ }^{2}$ similarly to those shown in Fig. 5.

The longitudinal polarizations probe the particle EDM, while the radial polarizations probe the axion dark matter and/or dark energy of vacuum (DM/DE). The positive and

\footnotetext{
${ }^{2}$ It is also possible to store beams with vertical polarizations, half of them directed upwards and the other half downwards as long as there are stored bunches with horizontal polarizations within the same storage time to indicate that the horizontal spin component is "frozen" relative to the momentum vector. This option can be explored to probe the EDM and the DM/DE cases simultaneously and to study additional systematic error sources.
}

negative helicities are mainly used to eliminate the polarimeter systematic errors, while the $\mathrm{CW}$ and $\mathrm{CCW}$ injections are used to eliminate systematic errors related to the background (unwanted) electromagnetic fields. Figure 6 shows the horizontal and vertical beta functions, indicating a highly symmetric beam envelope, a critical feature to reduce the sensitivity of the experiment on the latticeelement positioning errors. The beta function shows the stored beam envelope for each direction, which flips as the current polarity on the magnetic quadrupoles is flipped, providing one more tool to combat the systematic errors. Figure 7 shows the slip factor as a function of the quadrupole strength. The negative slip factor is required to establish equilibrium [12] between the three phase-space components of the stored beam due to intrabeam scattering, potentially allowing to increase the beam storage lifetime to more than $10^{4} \mathrm{~s}$. 

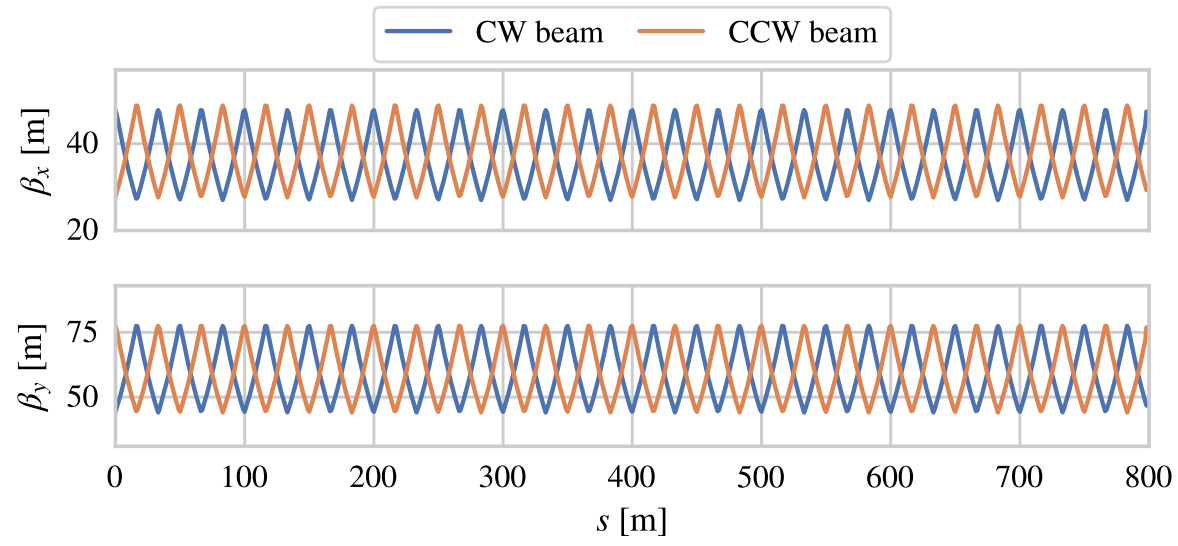

FIG. 6. The horizontal and vertical beta functions as a function of the longitudinal position around the ring. The two features, highly symmetric beta functions along the ring azimuth and quad polarity flipping, significantly supress the EDM and DM/DE systematics errors. While the beam envelope oscillates going around the ring, the center of the quads remain ideally at zero for all of them.

We have used high-precision simulation software with the required accuracy, similar or better than Ref. [34]. Field imperfections and lattice misalignments were included for the main systematic error assessments [35]. The interface between the fields and the straight section is a hard-edge approximation, with no fringe fields. The realistic fringe fields of infinitely high electric field plates with cylindrical geometry, as studied in Ref. [36], do not have a significant effect on particle beam/spin dynamics. The beam parameters of the ring lattice used here are given in Table I, with a main characteristic being that of the high symmetry of the lattice elements.

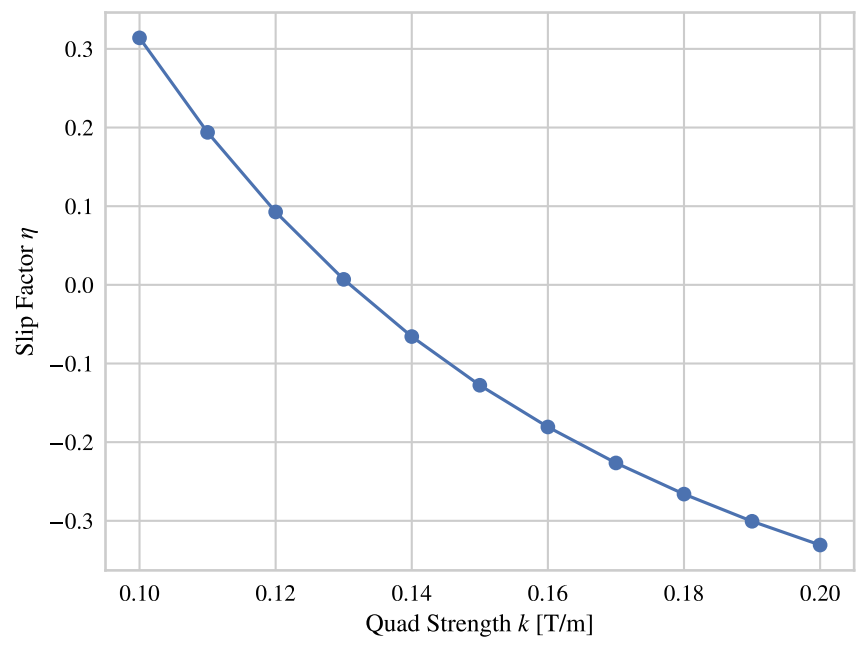

FIG. 7. The lattice slip factor as a function of the quadrupole strength. The negative slip factor puts the ring lattice below transition, an essential quality in having low intrabeam scattering and a stable storage for high beam intensities as required by the experiment [12].

\section{A. Backgrounds}

Our highly symmetric, hybrid ring lattice significantly relaxes the magnetic field shielding requirements as well as the magnetic quadrupole position tolerances. In this section we present the systematic errors and how we plan to eliminate them. Depending on the direction in which the spin is frozen, which also determines the physics the beam is sensitive to, the systematic errors are not necessarily the same. Running the experiment, with simultaneous storage of the bunched beams in all four different options, in both $\mathrm{CW}$ and $\mathrm{CCW}$ directions makes the experiment plausible. Table II shows the systematic errors for the EDM case and the remediation measures, while Table III shows the same for the radial polarization case. We will address the two cases separately below. The main systematic error source is the large $E$-field bending sections: either a vertical offset of

TABLE I. The lattice parameters for the storage-ring proton EDM experiment.

\begin{tabular}{|c|c|c|}
\hline Parameter & Magnitude & Description \\
\hline$p_{0}$ & $0.71 \mathrm{GeV} / c$ & Magic momentum \\
\hline$\beta$ & 0.59 & $=v / c$, the particle speed \\
\hline$R_{0}$ & $95.5 \mathrm{~m}$ & Deflector radius \\
\hline$C$ & $800 \mathrm{~m}$ & Ring circumference \\
\hline$f_{c}$ & $0.22 \mathrm{MHz}$ & Cyclotron frequency \\
\hline$f_{x}$ & $0.51 \mathrm{MHz}$ & Horizontal betatron frequency \\
\hline$Q_{x}$ & 2.3 & Horizontal betatron tune \\
\hline$f_{y}$ & $0.49 \mathrm{MHz}$ & Vertical betatron frequency \\
\hline$Q_{y}$ & 2.2 & Vertical betatron tune \\
\hline$E_{0}$ & $4.4 \mathrm{MV} / \mathrm{m}$ & Deflector electric field \\
\hline$k$ & $0.2 \mathrm{~T} / \mathrm{m}$ & Quadrupole strength \\
\hline$L_{\text {quad }}$ & $40 \mathrm{~cm}$ & Quadrupole length \\
\hline$L_{\text {str }}$ & $4.6 \mathrm{~m}$ & Straight section length (incl. quad.) \\
\hline$N$ & 48 & Number of cells \\
\hline
\end{tabular}


TABLE II. Main systematic errors and their remediation when hybrid fields (electric bending and magnetic focusing) are used for the EDM (longitudinal polarization) case. S-BPM are the SQUID-based beam position monitors; see below.

\begin{tabular}{|c|c|}
\hline Effect & Remediation \\
\hline Radial $B$ field. & Magnetic focusing. \\
\hline $\begin{array}{l}\text { Unwanted vertical forces when other than magnetic focusing is } \\
\text { present. }\end{array}$ & $\begin{array}{l}\text { Vary the magnetic focusing strength and fit for the dc offset in } \\
\text { the vertical precession rate [15]. }\end{array}$ \\
\hline Dipole vertical $E$ fields. & Cancel exactly with $\mathrm{CW}$ and $\mathrm{CCW}$ beam storage. \\
\hline Quadrupole $E$ field in the electric bending sections. & $\begin{array}{l}\text { Probe it by locally splitting the counterrotating beams and } \\
\text { cancel it with trim } E \text { fields. Finally, keep the counterrotating } \\
\text { beams at the same position to S-BPM resolution. }\end{array}$ \\
\hline Corrugated (nonplanar) orbit. & $\begin{array}{l}\text { Minimize effect with symmetric lattice design. Finally, keep the } \\
\text { CR beams at same position, at the electric field bending } \\
\text { sections, using beam-based alignment. }\end{array}$ \\
\hline Longitudinal $B$ field. & Small effect. \\
\hline Geometrical phase effect due to lattice element imperfections. & $\begin{array}{l}\text { Equivalent to a spin resonance due to lattice element } \\
\text { imperfections. Magnetic quadrupoles: beam-based alignment } \\
\text { to } 1 \mu \mathrm{m} \text { rms. E-field sections: absolute beam position } \\
\text { monitors to }<0.1 \mathrm{~mm} \text {. }\end{array}$ \\
\hline Geometrical phase effect due to external magnetic fields. & $\begin{array}{l}\text { Equivalent to a spin resonance due to external magnetic } \\
\text { interference coupled with electric field bending section } \\
\text { misplacement }[26,37] \text {. When the local spin effects are kept } \\
\text { below } 1 \mathrm{nT} B \text { field equivalent, the effect is negligible even for } \\
\text { one-directional (CW or CCW only) storage. }\end{array}$ \\
\hline RF cavity vertical and horizontal misalignment. & $\begin{array}{l}\text { Vary the longitudinal lattice impedance to probe the effect of the } \\
\text { cavity's vertical and horizontal angular misalignments. The } \\
\text { vertical and horizontal offsets are much smaller effects. }\end{array}$ \\
\hline
\end{tabular}

TABLE III. Main systematic errors and their remediation when hybrid fields (electric bending and magnetic focusing) are used for the DM/DE, probing (pseudo)scalar fields, (radial polarization) case.

\begin{tabular}{|c|c|}
\hline Effect & Remediation \\
\hline Radial $B$ field. & Small effect. \\
\hline $\begin{array}{l}\text { Unwanted vertical forces when other than magnetic focusing is } \\
\text { present. }\end{array}$ & Small effect. \\
\hline Dipole vertical $E$ fields. & Small effect. \\
\hline Quadrupole $E$ field in the electric bending sections. & Small effect. \\
\hline Corrugated (nonplanar) orbit. & $\begin{array}{l}\text { Minimize effect with symmetric lattice design. Finally, keep the } \\
\text { stored beams at zero average vertical angle when integrating } \\
\text { over the electric field bending sections. }\end{array}$ \\
\hline Longitudinal $B$ field. & $\begin{array}{l}\text { The } \mathrm{CW} \text { and } \mathrm{CCW} \text { stored proton spins rotate in the same } \\
\text { direction, while the (pseudo)scalar fields rotate them in } \\
\text { opposite directions. }\end{array}$ \\
\hline Geometrical phase effect due to lattice elements imperfections. & $\begin{array}{l}\text { Equivalent to a spin resonance due to lattice element } \\
\text { imperfections. Magnetic quadrupoles: beam-based alignment } \\
\text { to } 1 \mu \mathrm{m} \text { rms. E-field sections: absolute beam position } \\
\text { monitors to }<0.01 \mathrm{~mm} \text { per injection. }\end{array}$ \\
\hline Geometrical phase effect due to external magnetic fields. & $\begin{array}{l}\text { Equivalent to a spin resonance due to external magnetic } \\
\text { interference coupled with electric field bending section } \\
\text { misplacement }[26,37] \text {. When the local spin effects are kept } \\
\text { below } 1 \mathrm{nT} B \text { field equivalent, the effect is negligible even for } \\
\text { one-directional (CW or CCW only) storage. In this } \\
\text { polarization case, the relevant fields and lattice } \\
\text { misplacements may be in a different direction than in the } \\
\text { previous table. }\end{array}$ \\
\hline RF cavity vertical and horizontal misalignment. & Small effect. \\
\hline
\end{tabular}


the counterrotating beams (for the EDM case) or different vertical angles of the counterrotating beams (DM/DE case).

The effect of the external magnetic field is effectively shielded by the magnetic quadrupoles. When magnetic focusing is used, the vertical $E$ field is a major potential systematic error [38], since in its own rest frame it is partially converted into a radial magnetic field precessing the spin vertically. However, the virtue of alternate magnetic focusing is that it also allows for simultaneous CW and $\mathrm{CCW}$ storage to completely cancel the dipole vertical electric field effect. Vertical dipole $E$ field originating somewhere in the ring is canceled by a single trim dipole $E$-field placed anywhere along the beam orbit for both CW and CCW directions. However, if the vertical $E$ field is not uniform and the counterrotating beams do not map out the same field on average, this will create a potential systematic error source, which can also be the dominant one in this case.

In the radial polarization case, the main systematic error source is the average vertical velocity vector, integrated inside all the electric bending field sections. In this case, the vertical velocity at a radial $E$-field location creates a restframe longitudinal $B$ field, which will rotate the spin into the vertical direction and it is similar to the DM/DE effect for the $\mathrm{CW}$ and $\mathrm{CCW}$ directions. Therefore, any source of this systematic error needs to be symmetric enough so that it cancels when integrated around the ring within a single direction. In a highly symmetric ring lattice, like the one assumed here, the distortion of the closed orbit, caused by the position error of the magnetic quads and of the bending sections, is symmetric and will cancel to a high degree when integrating around the ring within a single direction. On the other hand, any longitudinal magnetic field, caused by a possible nonzero current going through the ring in the vertical direction, would also cancel. The $\mathrm{CW}$ and $\mathrm{CCW}$ beams will have their spins rotated in the same direction as opposed to the DM/DE effect.

\section{B. Ring lattice design}

The tools we have at our disposal to minimize and eliminate the potential systematic errors are the following:

(1) Design a symmetric lattice to minimize the effect of the lattice-element positioning errors.

(2) Shield the external magnetic field anywhere in the storage ring to below $100 \mathrm{nT}$. This can be easily achieved by using an array of flux-gate magnetometers and shimming the residual field using an array of current wires. The aim for the low azimuthal harmonics, i.e., $N=1,2, \ldots, 10$, of the $B$ field is to reach below $1 \mathrm{nT}$ amplitude.

(3) The SQUID-based beam-position monitors (SBPMs) developed at IBS-CAPP in collaboration with KRISS in Korea utilizing special, low-temperature superconducting SQUID gradiometers, have been shown to exhibit $10 \mathrm{~nm} / \sqrt{\mathrm{Hz}}$ [15], i.e., every
$100 \mathrm{~s}$ they can provide $1 \mathrm{~nm}$ beam separation resolution. The S-BPMs will be used to reduce the external $B$ field and the magnetic quadrupole position tolerance by modulating the vertical tune and by using beam-based alignment $[39,40]$.

\section{Position tolerances of ring elements}

Here, we address the main systematic errors in both the $\mathrm{EDM}$ and DM/DE cases in more detail, their origin, and the way to eliminate them. The main issue in the EDM case arises from the subtle effect that there might be a quadrupole $E$ field present, most likely at the electric bending sections, while there is a radial $B$ field present somewhere in the ring. A radial $B$ field of any order will inevitably split the vertical position of the counterrotating beams, which again will mean that the counterrotating beams will sense vertical electric fields of opposite direction or at least not of the same amplitude and therefore, the cancellation will only be partial. Only same-direction/same-amplitude vertical $E$ fields cancel with $\mathrm{CW}$ and $\mathrm{CCW}$ beams, meaning that the counterrotating beams will cancel the vertical dipole electric field (uniform field), but not higher order when the beams do not overlap at the required precision. The unknown in this case is the vertical $E$-field focusing index in the $E$-bending sections, which needs to be designed and installed as well as possible. Technically, it is possible to mechanically align the plates enough to achieve an average field focusing index of 1.1 (requiring an average alignment of order $10 \mu \mathrm{m}$ between the two plates and a similar plate flatness over $20 \mathrm{~cm}$ vertically, integrated over a $3 \mathrm{~m}$ azimuthal length of the plates). We plan to use a local radial $B$ field to separate the two counterrotating beams by as much as $1 \mathrm{~mm}$ locally at each bending section, and apply a trim quadrupole $E$ field at the specific $E$-bending section to eliminate the vertical nonuniformity of $E$ fields to the needed accuracy. We will be able to reduce the unwanted electric quadrupole component of the $E$-field sections to well below the 1.001 level, so that a local separation of the counterrotating beams of less than $0.1 \mu \mathrm{m}$ is adequate. The issue is to keep the plate alignment stability to better than $0.1 \mu \mathrm{m}$ over $10^{3} \mathrm{~s}$, which should not be a major challenge.

Our S-BPMs have an estimated resolution of $10 \mathrm{~nm} /$ $\sqrt{\mathrm{Hz}}$ [15], but even a resolution of $0.1 \mu \mathrm{m}$ within a fill of $10^{3} \mathrm{~s}$ duration of the beam separation between $\mathrm{CW}$ and $\mathrm{CCW}$ directions, would be adequate. Assuming a standard deviation in the vertical electric field focusing index in the $E$-bending sections of 1.001 would be adequate to reduce the main systematic errors below the statistical sensitivity. The total experiment duration is assumed to be $4 \times 10^{7} \mathrm{~s}$, meaning approximately $4 \times 10^{4}$ fills, resulting in an unwanted maximum vertical spin precession rate of $\approx 0.75 \mathrm{nrad} / \mathrm{s}$, corresponding to an EDM of $0.75 \times$ $10^{-29} e \cdot \mathrm{cm}$ in the same ring. A $10 \mu \mathrm{m}$ resolution in the position of the quadrupoles has been demonstrated using 
beam-based alignment in dc mode in hadronic storage rings [40], while the S-BPMs and the method we have developed to probe the average separation of the counterrotating (CR) beams, have much better resolution than that.

Next, we address the radial polarization case. Here, as stated previously, it is the average vertical velocity, when integrated over all electric bending sections, that dominates the systematic error source. Systematic error studies with high-precision simulations indicate that for a symmetric ring with the magnetic quadrupole positionerror randomly distributed with a standard deviation of $1 \mu \mathrm{m}$, and a quadrupole component in the electric field bending sections with a sigma of 1.001 , the systematic error is below the aimed statistical error of $10^{-29} e \cdot \mathrm{cm}$.

Constructing and setting up a ring that can deliver the necessary sensitivity requires a thorough study of all possible systematic error sources. Those listed in Tables II and III are the main ones regarding the spin-related systematic errors, while the systematic errors related to the polarimeter detector are adequately addressed by simultaneously storing positive- and negative-helicity beams [32].

\section{Construction, commission, and running the experiment}

The sequence of constructing, commissioning and running the experiment is as follows:

(1) The ring lattice is symmetric, i.e., the whole ring is designed to be approximately $800 \mathrm{~m}$ long in circumference, consisting of 48 identical units of length $16.67 \mathrm{~m}$ each. Each unit consists of a bending segment made of electric field plates of length $12 \mathrm{~m}$ each and one magnetic quadrupole of length $0.4 \mathrm{~m}$ capable of delivering a magnetic field of at least $0.2 \mathrm{~T} / \mathrm{m}$ made completely of wires.

(2) In addition, there is one RF cavity for beam bunching, two beam-injection points with kickers (if they are magnetic they need to be made out of wires), and one RF dipole to rotate the spin from vertical at injection to the required horizontal direction. Furthermore, there are a couple of polarimeter locations equipped with the required detectors to observe the spin precession rate in the horizontal and vertical directions for both $\mathrm{CW}$ and $\mathrm{CCW}$ stored beams. Other essential elements, e.g., BPMs based on sensing either the beam electric field or the beam generated magnetic field are also present as needed. There are S-BPMs, just before and after each electric field bending section to probe the separation of the $\mathrm{CR}$ beams and possibly paired with button BPMs for electric field sensing, to probe the transverse position of the CR beams.

(3) The electric field plates are separated by $3 \mathrm{~cm}$ of vertical, metallic plates of cylindrical geometry made as parallel as possible. In the rest of the ring there are parallel thin plates shielding the proton beams from vertical electrical forces caused by the induced charges on the ground plates. Since the beams are only moderately relativistic, we need to design the electric field plate geometry to minimize the lattice impedance and also to be able to vary it for systematic error studies.

(4) The vacuum chamber should be made out of bakeable aluminum, capable of reaching below $10^{-10}$ Torr in order to minimize beam-gas scattering. The aluminum and all materials near the beam should be of low magnetization (probably notrecycled aluminum) in order to keep the permanent-stray magnetic field in the storage region below $100 \mathrm{nT}$ at all locations.

(5) Construct the ring lattice with $1 \mathrm{~mm}$ position mechanical tolerance. Vertically (critical), using water tubes, minimize the lattice corrugation to below $100 \mu \mathrm{m}$, with special attention to keeping the low-azimuthal harmonics below an amplitude of $10 \mu \mathrm{m}[41,42]$.

(6) Install a current-carrying wire network to be able to cancel unwanted external magnetic fields paired with flux-gate magnetometers with enough resolution to achieve below $1 \mathrm{nT}$ for the low-azimuthal harmonics of the magnetic field amplitude in all directions.

(7) Inject into the ring both $\mathrm{CW}$ and $\mathrm{CCW}$ beams with vertical polarization direction (stable spin direction), and let the beams debunch without powering the RF cavity. Then, power the RF cavity slowly and rebunch the beam into the required number of bunches.

(8) Apply the RF solenoid to precess the spin from vertical into the four spin directions (forward, backward, radially outward and radially inward) for both CR directions.

(9) Tune the RF cavity frequency, so that the particle spins precess in the horizontal plane and measure the beam polarization value. Next, freeze the spin in the desired direction by using feedback signals from the polarimeters. If a single RF frequency does not simultaneously freeze the horizontal spin precessions of the CR beams, then apply a small vertical dipole magnetic field to freeze both of them.

(10) The vertical alignment specs of the electric field plates are very strict, and difficult to achieve mechanically. However, in the hybrid ring and with simultaneous CW and CCW beam storage the effect is canceled in a straightforward way by placing a trim vertical dipole $E$ field anywhere in the ring. The amplitude of the trim dipole $E$ field is adjusted by zeroing the vertical spin precession rate of either the $\mathrm{CW}$ - or CCW-going beam.

(11) Apply beam-based alignment with a detector resolution better than $10 \mu \mathrm{m}$, while keeping the low-azimuthal harmonics, i.e., $N=1,2, \ldots, 10$, of 
the lattice element deviations from the ideal location at better than $0.1 \mu \mathrm{m}$.

(12) Minimize the separation of the CW and CCW beams before and after the electric bending sections, which is the main source of systematic errors.

(13) Increase the lattice impedance to increase the sensitivity to the RF cavity horizontal and vertical angle misalignments. Rotate the RF cavity to reduce the effect below the statistical sensitivity with one injection and then return the ring lattice impedance to minimum.

(14) Increase the separation of the CR beams to $1 \mathrm{~mm}$ locally at one electric field bending section at a time. This can be easily achieved by using radial magnetic fields before and after the electric bending sections. This effect increases the sensitivity of the experiment to the electric quadrupole component, which can be trimmed out to the statistical sensitivity level. Repeat for all 48 plate sections and then return to the minimum beam separation.

(15) Run the experiment to probe the proton EDM and the DM/DE of vacuum with full sensitivity.

Before constructing the ring, a test section of one section of the ring will be constructed and tested to provide information regarding compatibility issues at the integration stage. After that, constructing the ring should take less than 2 years to accomplish, with the commissioning and systematic error studies taking less than 6 months to accomplish. The experiment should take about 4 years of running to accumulate the required statistics for $10^{-29} e$. $\mathrm{cm}$ sensitivity on the proton EDM and comparable sensitivity for the DM/DE.

\section{DISCUSSION}

Storage rings are competitive with atomic comagnetometer spin precession experiments in searching for interactions between protons and cosmic pseudoscalar and magnetic dipole vector backgrounds. This is because the relativistic velocity between the protons and the background enhances the velocity-dependent spin precession, compensating for the much larger shot noise in storage ring experiments in comparison to atomic comagnetometer experiments. However, background vector fields that interact through electric dipole couplings are harder to probe using storage ring experiments. This is because the electric dipole precession is not amplified by the relativistic motion of the protons. Thus, due to the much higher shot noise in storage ring experiments in comparison to atomic comagnetometer experiments, the latter will be significantly more sensitive to such vector interactions. Storage ring experiments to search for Lorentz-violating cosmic backgrounds are thus complementary to atomic comagnetometer searches for such backgrounds, allowing us to even distinguish the microphysics of a signal in such experiments.

It is also important to highlight that the search for cosmic backgrounds using storage ring experiments can use the same hardware as the setup used to search for the electric dipole moment of nucleons. The major difference in terms of the operation of the device concerns the orientation of the nucleon spin relative to the direction of motion of the proton around the ring. Implementation of this change should not be unduly onerous. While the backgrounds for an electric dipole moment search are different from those that would affect this cosmic search, the technologies developed to implement the electric dipole moment search can be directly applied to mitigate the corresponding backgrounds for the cosmic search. Thus, this laboratory search for dark energy and dark matter complements the use of storage rings to search for nucleon electric dipole moments.

\section{ACKNOWLEDGMENTS}

We would like to thank Savas Dimopoulos and Roni Harnik for discussions. P. W. G. was supported by DOE Grant No. DE-SC0012012, by NSF Grant No. PHY1720397, the Heising-Simons Foundation Grants No. 2015-037 and 2018-0765, DOE HEP QuantISED Award No. 100495, and the Gordon and Betty Moore Foundation Grant No. GBMF7946. S. R. was supported in part by the NSF under Grants No. PHY-1818899 and No. PHY-1638509, the Simons Foundation Grant No. 378243 and the Heising-Simons Foundation Grant No. 2015-038. S. H., Z. O. and Y. K. S. were supported by IBS-R017-D1-2020-a00 of the Republic of Korea. D. E. K. was supported in part by the NSF under Grant No. PHY1818899.
[1] M. Pospelov and M. Romalis, Phys. Today 57, No. 7, 40 (2004).

[2] M. V. Romalis and R. R. Caldwell, arXiv:1302.1579.

[3] G. W. Bennett et al., Phys. Rev. Lett. 100, 091602 (2008).
[4] J. M. Brown, S. J. Smullin, T. W. Kornack, and M. V. Romalis, Phys. Rev. Lett. 105, 151604 (2010).

[5] P. W. Graham, D. E. Kaplan, J. Mardon, S. Rajendran, W. A. Terrano, L. Trahms, and T. Wilkason, Phys. Rev. D 97, 055006 (2018). 
[6] C. Gemmel et al., Phys. Rev. D 82, 111901 (2010).

[7] P. W. Graham and S. Rajendran, Phys. Rev. D 88, 035023 (2013).

[8] P. W. Graham, D. E. Kaplan, and S. Rajendran, Phys. Rev. D 100, 015048 (2019).

[9] P. W. Graham, D. E. Kaplan, and S. Rajendran, Phys. Rev. D 97, 044003 (2018).

[10] D. Budker, P. W. Graham, M. Ledbetter, S. Rajendran, and A. Sushkov, Phys. Rev. X 4, 021030 (2014).

[11] F. J. M. Farley, K. Jungmann, J. P. Miller, W. M. Morse, Y. F. Orlov, B. L. Roberts, Y. K. Semertzidis, A. Silenko, and E. J. Stephenson, Phys. Rev. Lett. 93, 052001 (2004).

[12] V. Anastassopoulos et al., Rev. Sci. Instrum. 87, 115116 (2016).

[13] J. Bailey et al., Nucl. Phys. B150, 1 (1979).

[14] N. Aghanim et al. (Planck Collaboration), Astron. Astrophys. 641, A6 (2020).

[15] S. Hacıömeroğlu and Y. K. Semertzidis, Phys. Rev. Accel. Beams 22, 034001 (2019).

[16] J. H. Chang, R. Essig, and S. D. McDermott, J. High Energy Phys. 09 (2018) 051.

[17] M. Tanabashi et al. (Particle Data Group), Phys. Rev. D 98, 030001 (2018) and 2019 update.

[18] K. Hamaguchi, N. Nagata, K. Yanagi, and J. Zheng, Phys. Rev. D 98, 103015 (2018).

[19] A. Sedrakian, Phys. Rev. D 93, 065044 (2016).

[20] R. Bollig, W. DeRocco, P. W. Graham, and H. T. Janka, Phys. Rev. Lett. 125, 051104 (2020).

[21] S. Andreas, O. Lebedev, S. Ramos-Sanchez, and A. Ringwald, J. High Energy Phys. 08 (2010) 003.

[22] C. Brust, D. E. Kaplan, and M. T. Walters, J. High Energy Phys. 12 (2013) 058.

[23] F. D'Eramo, R. Z. Ferreira, A. Notari, and J. L. Bernal, J. Cosmol. Astropart. Phys. 11 (2018) 014.

[24] D. Baumann, D. Green, and B. Wallisch, Phys. Rev. Lett. 117, 171301 (2016).
[25] S. Haciömeroğlu, D. Kawall, Y.-H. Lee, A. Matlashov, Z. Omarov, and Y. K. Semertzidis, Proc. Sci., ICHEP2018 (2019) 279.

[26] S. Haciömeroğlu, Y. Orlov, and Y. K. Semertzidis, Nucl. Instrum. Methods Phys. Res., Sect. A 927, 262 (2019).

[27] Y. Orlov, E. Flanagan, and Y. Semertzidis, Phys. Lett. A 376, 2822 (2012).

[28] P. Benati etal., Phys. Rev. STAccel. Beams 15, 124202 (2012).

[29] P. Benati et al., Phys. Rev. ST Accel. Beams 16, 049901 (2013).

[30] Y. Orlov, arXiv:1504.07304.

[31] Y. Orlov, arXiv:1506.02069.

[32] N. P. M. Brantjes et al., Nucl. Instrum. Methods Phys. Res., Sect. A 664, 49 (2012).

[33] G. Guidoboni et al., Phys. Rev. Lett. 117, 054801 (2016).

[34] E. M. Metodiev et al., Nucl. Instrum. Methods Phys. Res., Sect. A 797, 311 (2015).

[35] Z. Omarov, S. Haciomeroglu, V. Lebedev, W. Morse, A. J. Silenko, E. J. Stephenson, and Y. K. Semertzidis, arXiv: 2007.10332.

[36] E. M. Metodiev, K. L. Huang, Y. K. Semertzidis, and W. M. Morse, Phys. Rev. ST Accel. Beams 17, 074002 (2014).

[37] S. Haciömeroğlu and Y. K. Semertzidis, arXiv:1709.01208.

[38] V. Anastassopoulos et al., A proposal to measure the proton electric dipole moment with $10^{-29} e \cdot \mathrm{cm}$ sensitivity by the Storage Ring EDM Collaboration, 2011, https://www.bnl .gov/edm/files/pdf/Proton_EDM_proposal_20111027_final .pdf.

[39] P. Tenenbaum and T. O. Raubenheimer, Phys. Rev. ST Accel. Beams 3, 052801 (2000).

[40] T. Wagner et al., Hyperfine Interact. 239, 61 (2018).

[41] B. Baklakov, T. Bolshakov, A. Chupyra, A. Erokhin, P. Lebedev, V. Parkhomchuk, S. Singatulin, J. Lach, and V. Shiltsev, Phys. Rev. ST Accel. Beams 1, 031001 (1998).

[42] V. Shiltsev et al., https://icfa-nanobeam.web.cern.ch/icfananobeam/paper/shiltsev_Tev_vibrations_v2.pdf. 